\title{
A qualidade da atenção primária e o manejo do diabetes mellitus
}

\author{
Primary health care quality and diabetes care \\ La calidad de la atención primaria y el manejo de la diabetes mellitus
}

\begin{abstract}
Marcelo Rodrigues Gonçalves. Universidade Federal do Rio Grande do Sul (UFRGS). Porto Alegre, RS, Brasil. marcelorog@gmail.com (Autor correspondente) Erno Harzheim. Universidade Federal do Rio Grande do Sul (UFRGS). Porto Alegre, RS, Brasil. ernoharz@terra.com.br

Aline do Amaral Zils. Universidade Federal do Rio Grande do Sul (UFRGS). Porto Alegre, RS, Brasil. aline.zils@gmail.com

Bruce Bartholow Duncan. Universidade Federal do Rio Grande do Sul (UFRGS). Porto Alegre, RS, Brasil. bbduncan@ufrgs.br
\end{abstract}

\section{Resumo}

Objetivos: Investigar a associação entre qualidade da atenção primária à saúde (APS) e manejo do diabetes mellitus (DM) nos adultos portadores de DM adscritos aos serviços de APS em Porto Alegre, Brasil. Métodos: Estudo transversal de base populacional, com adultos portadores de DM. A qualidade da APS foi aferida pelo Primary Care Assesment Tool (PCATool-Brasil). A análise estatística foi realizada por regressão de Poisson com variação robusta. Resultados: Dos 3.014 entrevistados, 205 (6,8\%) eram portadores de DM, com predomínio do sexo feminino $(64,4 \%)$ e cor branca $(68,3 \%)$. Os portadores de diabetes classificados como de Alto Escore apresentavam prevalência maior, tanto no tempo de doença (10,9 vs 8,4 anos, $p=0,03)$ quanto em complicações do diabetes $(73,9 \%$ vs $58,8 \%, p=0,02)$. A proporção de entrevistados com ótimo controle glicêmico não diferenciou entre os grupos $(31,7 \%$ vs $38 \%, p=0,3)$. Na análise multivariável, serviços com Alto Escore à APS apresentaram melhor perfil de cuidados para a prevenção das principais comorbidades. Os manejos que diferiram estatisticamente foram a aferição da pressão arterial (RP=1,07; IC95\% 1,01-1,14); solicitação de perfil lipídico ( $R P=1,23$; IC95\% 1,09-1,39); orientação de atividade física ( $R P=1,50$; IC95\% 1,21-1,86); exame dos pés (RP=2,08; IC95\% 1,54-2,81); e orientações de cuidados com os pés ( $\mathrm{RP}=2,02 ;$ IC95\% 1,56-2,61). Conclusão: Serviços com Alto Escore à APS demonstraram maior atenção à prevenção das complicações do diabetes e melhor cuidado aos pacientes com doença de maior complexidade, mas não diferiram significativamente dos demais serviços no controle glicêmico.

\section{Abstract}

Objective: To investigate the association between primary health care (PHC) quality and diabetes mellitus (DM) management in adult patients living within the catchment area of PHC services in Porto Alegre, Brazil. Methods: Cross-sectional, population-based study of adults reporting known diabetes. Quality of PHC was assessed through the Primary Care Assesment Tool (PCATool-Brazil). Statistical analyses were performed with Poisson regression with robust variance. Results: Of the 3,014 adults interviewed, 205 (6.8\%) reported having diabetes; of these, $64.4 \%$ were women and $68.3 \%$ were white. Regarding PHC score of the health service attended, people with diabetes that were classified with a high PHC score, presented longer duration of disease ( 10.9 vs 8.4 anos, $p=0.03$ ) and greater frequency of diabetes-related complications $(75.3 \%$ vs $58.8 \%, p=0.02)$. Regarding the proportion of respondents with good glycemic control, no significant difference between groups was found $(31.7 \% \mathrm{vs} 38 \%, p=0.3)$. In the multivariate analysis, services with a high PHC score presented a better profile of care for the prevention of the main comorbidities - greater blood pressure assessment $(P R=1.07$; Cl95\% 1.01-1.14), lipid profile request ( $P R=1.23$; Cl95\% 1.09-1.39), counseling for physical activity ( $P R=1.50 ; C l 95 \% 1.21-1.86)$, foot examination ( $\mathrm{PR}=2.08$; $\mathrm{Cl} 95 \%$ 1.54-2,81), and counseling for foot care ( $\mathrm{PR}=1.37$; Cl95\% 1.18-1.59). Conclusion: High PHC score services showed better performance in the management of diabetes and care for more complicated patients, but they did not differ significantly from lower PHC score services in terms of patients' glycemic control.

\section{Resumen}

Objetivos: Investigar la asociación entre la calidad de la atención primaria de la salud (APS) y el control de la diabetes mellitus (DM) en los adultos con DM adscritos a los servicios de APS en Porto Alegre, Brasil. Métodos: Estudio transversal de base poblacional con adultos portadores de DM. La calidad de la APS se midió con la Herramienta de Evaluación de la Atención Primaria (PCATool-Brasil). El análisis estadístico se realizó mediante regresión de Poisson con varianza robusta. Resultados: De los 3.014 encuestados, 205 (6,8\%) pacientes tenían DM, con predominio del sexo femenino $(64,4 \%)$ y de la piel de color blanca (68,3\%). Los pacientes con diabetes clasificados con puntuaciones altas tenían mayor prevalencia, tanto en la duración de la enfermedad ( 10,9 vs. 8,4 años, $p=0,03)$ como en complicaciones de la diabetes $(73,9 \%$ vs. $58,8 \%, p=0,02)$. La proporción de encuestados con buen control glucémico no difirió entre los grupos ( $31,7 \%$ vs $38 \%, p=0,3)$. En el análisis multivariado, servicios con altas puntuaciones en la APS mostraron mejor perfil de atención para la prevención de las principales enfermedades concomitantes. Los manejos que difirieron significativamente fueron: medición de la presión arterial ( $R P=1,07$, IC95\% 1,01-1,14), solicitud de perfil de lípidos ( $R P=1,23$, IC95\%: 1,09-1,39), orientación de la actividad física $(\mathrm{RP}=1,50$, IC95\% 1,21-1,86), examen de los pies ( $\mathrm{RP}=2,08$, IC95\% 1,54-2,81) y orientaciones de la atención para los pies ( $\mathrm{RP}=2,02$, IC95\% 1,562,61). Conclusión: Servicios con una alta puntuación en la APS demostraron una mayor atención en la prevención de complicaciones de la diabetes y una mejor atención a los pacientes con enfermedad más compleja, pero no difirieron significativamente de los otros servicios en el control glucémico.

Como citar: Gonçalves MR, Harzheim E, Zils AA, Duncan BB. A qualidade da atenção primária e o manejo do diabetes mellitus. Rev Bras Med Fam Comunidade. 2013; 8(29):235-43. Disponível em: http://dx.doi.org/10.5712/rbmfc8(29)814
Palavras-chave: Atenção Primária à Saúde Diabetes Mellitus Pesquisa sobre Serviços de Saúde

Keywords: Primary Health Care Diabetes Mellitus Health Services Research

Palabras clave: Atención Primaria de Salud Diabetes Mellitus Investigación sobre Servicios de Salud

Fonte de financiamento: FAPERGS, CNPq e CAPES.

Parecer CEP: 2004367 (Propesq - UFRGS) aprovado em 13/01/2005.

Conflito de interesses: declaram não haver. Recebido em: 16/04/2013 Aprovado em: 20/08/2013 


\section{Introdução}

Desde que Lord Dawson, em 1920, esboçou pela primeira vez o conceito de regionalização do sistema de saúde, distinguindo três níveis de atenção ${ }^{1}$, até o marco teórico da moderna conceituação de atenção primária à saúde (APS), definida por Starfield ${ }^{2}$ por meio de seus atributos essenciais (acesso de primeiro contato do indivíduo com o sistema de saúde, longitudinalidade e integralidade nas açôes e coordenação dentro do sistema) e derivados (orientação familiar, comunitária e competência cultural), diversas pesquisas têm relacionado a maior extensão destes atributos com maior efetividade e equidade do cuidado à saúde ${ }^{3-5}$. Ao apresentar os atributos essenciais, um serviço de saúde pode ser considerado provedor de atenção primária ${ }^{6}$, sendo maior sua orientaçáo se estiverem presentes também os atributos derivados ${ }^{2,7,8}$.

O Brasil, assim como outros países de média e baixa renda, passa por um acelerado processo de transiçáo demográfica e epidemiológica, com uma mudança no perfil de morbimortalidade da população, identificado pela diminuição da mortalidade por doenças infecciosas e materno infantis e pelo aumento das doenças crônicas não transmissíveis (DCNT) e das mortes por causas externas ${ }^{9,10}$. Entre as DCNT, as doenças cardiovasculares representam hoje a maior causa de mortalidade no País, correspondendo a $28,7 \%$ dos óbitos em $2010^{11}$. Entre seus principais fatores de risco modificáveis encontra-se o diabetes mellitus (DM), que está associado a complicaçóes agudas e crônicas de vários órgáos e sistemas. O DM pode ser classificado em tipo 1, tipo 2, gestacional e outros menos prevalentes. Entretanto, do ponto de vista populacional, o DM do tipo 2 possui maior importância, pois, atualmente, representa até $90 \%$ dos casos identificados, apresenta incidência crescente e, consequentemente, impóe enorme carga econômica aos sistemas de saúde ${ }^{12,13}$. O Ministério da Saúde brasileiro busca priorizar açóes de prevenção e manejo do DM, inclusive como ação programática na Estratégia Saúde da Família (ESF) ${ }^{14}$. Associado a este fato, desde 1994, o Brasil busca reorganizar seu modelo assistencial por meio da ampliação de acesso aos serviços de APS, com a adoção da ESF. Hoje, a ESF cobre 54,8\% da populaçáo brasileira ${ }^{15}$. Entretanto, a maioria das açóes programáticas, assim como da produção científica associada à efetividade da APS e qualidade dos cuidados prestados, tem tido foco nos modelos de saúde materno infantis ${ }^{1,16}$. Nesse contexto, existe crescente interesse em indicadores como internaçóes por condiçóes sensíveis à APS (iCSAPS) e as próprias DCNT.

A atenção ao diabetes é complexa e envolve uma multiplicidade de aspectos que vão além do simples controle glicêmico ${ }^{13,17}$. Um vasto corpo de evidências apoia diversas intervençôes para melhorar os desfechos macro e microvasculares no DM, implementados de acordo com a disponibilidade dos recursos locais ${ }^{18}$. Segundo a Associação Americana de Diabetes (ADA) ${ }^{17}$, a incorporação dos cuidados padronizados para o DM tem sido subutilizada na maioria dos cenários clínicos, devido principalmente à fragmentação do atendimento, duplicidade de serviços, inadequação nas informaçóes clínicas e debilidade nos modelos para atendimento de doenças crônicas, e se resume na insuficiência da extensão de alguns dos principais atributos da APS $^{12}$. A maioria dos estudos sobre a efetividade do cuidado em DM investiga a associação com atributos individuais da APS (principalmente acesso e longitudinalidade), estratégias de organização dos serviços de saúde, locais de prestação dos cuidados ou tipo de médico que exerce o atendimento. Não foram encontrados estudos associando a força da orientação para APS com o processo de atenção nestes pacientes ${ }^{19-23}$. O presente estudo tem como objetivo principal investigar a associação entre a qualidade da APS (escores do Primary Care Assessment Tool - PCATool) e o manejo dos portadores de DM, adscritos aos serviços de APS no município de Porto Alegre.

\section{Métodos}

\section{Delineamento do estudo e amostragem}

Conduzimos um estudo transversal de base populacional no período de julho de 2006 a agosto de 2007 no município de Porto Alegre/RS, do qual participaram adultos maiores de 18 anos de idade, adscritos às áreas geográficas cobertas pela rede pública de APS do município ou pertencentes à listagem de pacientes maiores de 18 anos atendidos pelas Equipes de Saúde da Família de um plano de saúde privado (CASSI-RS). O critério de adscrição às áreas geográficas cobertas pela rede pública de APS foi utilizado apenas para a seleção dos participantes, sendo avaliados os serviços de saúde referidos como de uso preferencial pelos entrevistados. Estes serviços poderiam não ter enfoque em APS (consultórios particulares e 
emergências hospitalares) e/ou serem classificados como privados. Foram considerados portadores de diabetes os usuários que autorreferiram essa condição à pergunta "Algum médico já lhe disse que você tem diabetes?".

Este estudo é parte do projeto "Avaliação da qualidade do processo de atenção e da sua efetividade sobre a saúde do adulto no Programa Saúde da Família e em modelos alternativos na rede de atenção primária do município de Porto Alegre”24, com amostra total calculada em 3000 pessoas, por meio do pacote estatístico EPI-INFO 6.0, utilizando dados provenientes do estudo de validação da versão infantil do PCATool $^{25}$ e da literatura nacional sobre prevalência, reconhecimento e controle do $\mathrm{DM}^{13}$. O processo de amostragem dos domicílios foi por conglomerado (clusters), sendo estratificada por gerência distrital de saúde do município e distribuída proporcionalmente ao tamanho das populaçóes adscritas a cada unidade de saúde dos serviços de APS. Os parâmetros utilizados para este cálculo foram: nível de confiança de $95 \%$, poder estatístico de $80 \%$, precisão de 3\%, com fator de delineamento de 1,2 para correção do efeito cluster.

\section{Instrumentos, medidas e variáveis}

1. Questionários. Foram realizadas medidas com um questionário geral, estruturado, composto por 3 instrumentos distintos:

- Questionário estruturado com variáveis sociodemográficas, avaliação de saúde, satisfação, hábitos de vida e sobre o processo de atenção aos portadores de diabetes (orientaçôes preventivas, medicamentos utilizados, exame físico, entre outros);

- Questionário sobre a qualidade da APS: Instrumento de Avaliação da Atenção Primária, versão adultos do PCATool-Brasil ${ }^{26}$, desenvolvido por Starfield, que mede a presença e a extensão dos quatro atributos essenciais (Acesso [A], Longitudinalidade [L], Integralidade [I] e Coordenação [C]) e dos três atributos derivados (Orientação Familiar [OF], Orientação Comunitária [OC]) da APS e o grau de afiliação (GA) ao serviço de saúde, definido como a força da relação entre o usuário e o serviço/profissional de saúde). Este instrumento, que já foi validado no Brasil por nosso grupo de pesquisa, possibilita, por meio de respostas tipo Likert, construir escores específicos e gerais sobre a extensão dos atributos, além de avaliar e planejar ações no âmbito da APS, a partir da experiência dos usuários. São considerados serviços com Alto Escore de APS aqueles que obtiverem valor maior ou igual a 6,6 (tercil superior do escore). O escore final de cada um destes atributos é dado pela média das respostas de seus itens (perguntas). Alguns atributos são formados por subdimensões, como Acesso (Primeiro Contato [A1] e Utilização [A2]) e Integralidade (Serviços Recebidos [I1] e Serviços Disponíveis [I2]). A média entre os escores dos atributos essenciais e o grau de afiliação produz o Escore Essencial da APS (EE, ver fórmula a seguir), e a dos escores dos atributos derivados produz o Escore Derivado da APS (ED). A média de todos estes escores produz o Escore Geral de APS (EG, ver fórmula a seguir).

Para o cálculo do EE e EG, as fórmulas são:

$$
\begin{gathered}
\mathrm{EE}=\frac{\mathrm{GA}+\mathrm{A} 1+\mathrm{A} 2+\mathrm{L}+\mathrm{C}+\mathrm{I} 1+\mathrm{I} 2}{7} \\
\mathrm{EG}=\frac{\mathrm{GA}+\mathrm{A} 1+\mathrm{A} 2+\mathrm{L}+\mathrm{C}+\mathrm{I} 1+\mathrm{I} 2+\mathrm{OF}+\mathrm{OC}}{9}
\end{gathered}
$$

A transformação da escala tipo Likert de 1 a 4 para escores em escala de 0 a 10 foi realizada utilizando a seguinte fórmula: (escore obtido -1$) \times 10$, onde 4 é o escore máximo e 1 o mínimo.

2. Medidas antropométricas:

- Peso, medido por balanças portáteis (Techline ${ }^{\oplus}$, modelo BAL-180-CI); altura, medida por estadiômetros portáteis $\left(\right.$ Sanny $\left.{ }^{\circ}\right)$; e cintura e quadril, medidos com fita métrica inelástica;

- Avaliação da pressão arterial: medida em dois momentos durante a visita domiciliar, com intervalo mínimo de 3 minutos entre a $1^{\text {a }}$ e a $2^{\text {a }}$ medida, com os entrevistadores treinados segundo as normas da Organizaçáo Mundial da $S_{\text {Saúde }}{ }^{7}$. Todos os instrumentos foram calibrados periodicamente e aprovados pelo Instituto Nacional de Metrologia, Normalização e Qualidade Industrial (INMETRO). 
3. Medidas bioquímicas: hemoglobina glicada (HbAlc) nos pacientes que reconheceram sua condição de diabéticos. As amostras de sangue foram coletadas por meio de agendamento nas unidades de saúde amostradas, por um técnico de enfermagem de um laboratório privado contratado. As amostras de sangue foram processadas por meio de cromatografia líquida de alta performance (HPLC - Bio-Rad), de acordo com orientaçôes internacionais ${ }^{28}$.

4. Variáveis Dependentes (Desfechos). Entre as variáveis analisadas, ressaltamos, principalmente, as de manejo do DM (exame e orientações para cuidado dos pés, pressão arterial (PA) aferida na última consulta e solicitação de perfil lipídico, além das orientaçôes para prática de exercícios físicos e dieta saudável). Foram considerados portadores de DM com ótimo controle glicêmico aqueles com $\mathrm{HbA1c}<7,0 \%$, conforme a ADA e diretrizes nacionais ${ }^{13,17}$.

\section{Análise estatística}

A análise descritiva (da avaliação dos serviços de saúde, da satisfação e do estado de saúde da população) levou em consideração a estratégia de amostragem (clusters), utilizando o pacote estatístico STATA 9.0 , por meio do Método de Séries Lineares de Taylor (comandos svy). Os dados foram apresentados por suas médias e desvios padrão, intervalos de confiança e proporçóes, expressos de forma contínua e categórica. Para a comparaçáo das médias foi utilizado o teste t de Student e, para as proporções, o teste do quiquadrado. O nível de significância de 5\%, bicaudal, foi utilizado para todas as provas estatísticas. Para o controle de possíveis fatores de confusão, foi construído um modelo teórico hierárquico (Figura 1) para o ajuste das variáveis dependentes (processo de atençáo ao DM). O modelo de Poisson, com variaçáo robusta para amostras por conglomerado, foi utilizado para análise multivariável. A escolha deste tipo de regressão baseou-se na alta prevalência dos desfechos medidos (>10\%) e para obtenção de estimativas da Razão de Prevalência (RP) com maior precisão nos intervalos de confiança (IC) ${ }^{29}$. A entrada das variáveis deu-se de acordo com o modelo teórico proposto, permanecendo aquelas com $\mathrm{p}<0,20$, devido à possibilidade de confundimento.

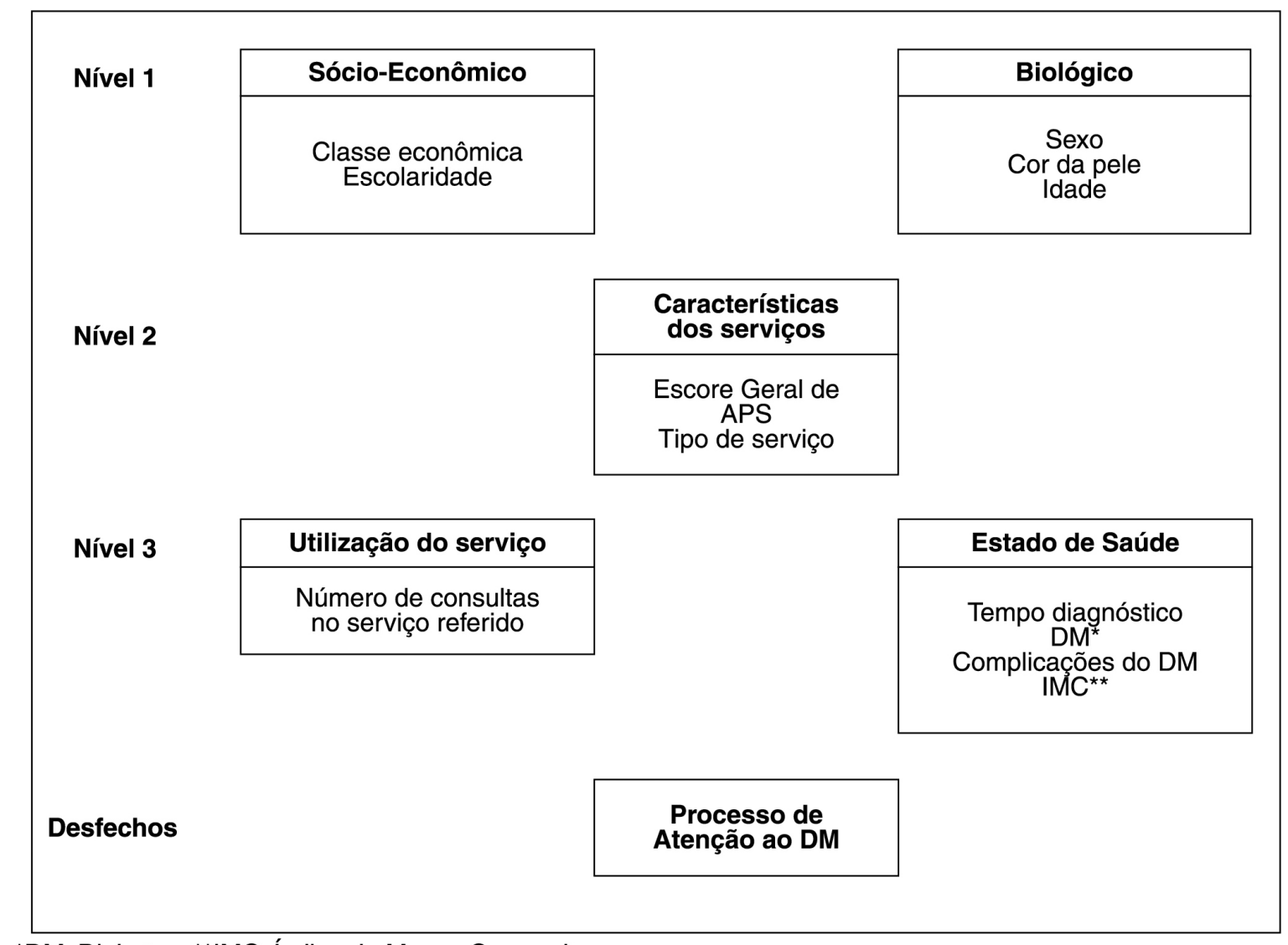

*DM: Diabetes; **IMC: Î́ndice de Massa Corporal.

Figura 1. Modelo teórico hierárquico para processo de atenção ao DM. 


\section{Aspectos éticos}

Este estudo foi aprovado pelo Comitê de Ética em Pesquisa da UFRGS, do Grupo Hospitalar Conceição, da Secretaria Municipal de Saúde de Porto Alegre, do Hospital de Clínicas de Porto Alegre (HCPA) e da Escola de Saúde Pública/RS. Todos os entrevistados, depois de informados e esclarecidos sobre os objetivos do estudo, assinaram o Termo de Consentimento Livre e Esclarecido (TCLE). Todos os indivíduos identificados pela primeira vez como portadores de HAS, assim como os portadores de HAS e DM não controlados adequadamente foram encaminhados para avaliação e acompanhamento ambulatorial em seus serviços de saúde.

\section{Resultados}

Dos 3.014 entrevistados no projeto "Avaliação da qualidade do processo de atenção e da sua efetividade sobre a saúde do adulto no Programa Saúde da Família e em modelos alternativos na rede de atenção primária do município de Porto Alegre", foram identificados 213 que se reconheciam como portadores de DM. Depois da coleta da HbA1c (185 entrevistados), oito foram descartados por apresentarem níveis $<6,0$, sem estarem em uso de hipoglicemiantes ou adotando medidas dietéticas, permanecendo um total de 205 (6,8\%) participantes. Dos usuários que não realizaram a coleta do exame, 9 foram recusas e 20 foram considerados perdas, após 3 tentativas de contato sem resposta.

Em relação ao grau de orientação à APS dos serviços de saúde, os portadores de DM classificados como de Alto Escore (Tabela 1) apresentavam prevalência significativamente maior, tanto no tempo de doença $(10,9$ vs 8,4 anos, $\mathrm{p}=0,03)$ quanto

Tabela 1. Características sociodemográficas e do estado de saúde dos portadores de diabetes classificados com Alto e Baixo Escore Geral da APS. Porto Alegre, 2007.

\begin{tabular}{|c|c|c|c|}
\hline Características & Alto Escore Geral da APS $(\geq 6,6)$ & Baixo Escore Geral da APS $(<6,6)$ & \multirow{2}{*}{$p^{*}$} \\
\hline Sociodemográficas (n) & n/média (\%/IC 95\%) & n/média (\%/lC 95\%) & \\
\hline Idade em anos & $60,4(55,8-65,1)$ & $61,2(57,3-65,1)$ & 0,7 \\
\hline Sexo & & & 0,4 \\
\hline Masculino & $22(31,9 \%)$ & $51(37,5 \%)$ & \\
\hline Feminino & $47(68,1 \%)$ & $85(62,5 \%)$ & \\
\hline Escolaridade (anos completos) & $6,98(5,52-8,45)$ & $7,85(6,76-8,93)$ & 0,1 \\
\hline Cor da Pele & & & 0,9 \\
\hline Branca & $47(68,1 \%)$ & $93(68,4 \%)$ & \\
\hline Não Branca & $22(31,9)$ & $43(31,6 \%)$ & \\
\hline Classe Econômica (ABIPEME) & & & 0,1 \\
\hline$A-B$ & $24(34,8 \%)$ & $61(44,8 \%)$ & \\
\hline C-D-E & $45(65,2 \%)$ & $75(55,1 \%)$ & \\
\hline Renda per capita - R\$ (200) & $754,4(397,2-1111,6)$ & $1129,6(595,6-1663,5)$ & 0,01 \\
\hline \multicolumn{4}{|l|}{ Estado de Saúde (n) } \\
\hline HbA1c \% ( $(n=176)$ & $8,27(7,68-8,86)$ & $8,01(7,53-8,49)$ & 0,2 \\
\hline Proporção DM com HbA1c < 7\% e Alto Escore APS ( $n=185)$ & $20(31,7 \%)$ & $43(38 \%)$ & 0,3 \\
\hline Tempo de diagnóstico de DM em anos & $10,9(8,6-13,2)$ & $8,37(7,2-9,5)$ & 0,03 \\
\hline Número de internações por DM ao longo da vida & $2,6(1,6-3,7)$ & $3,6(1,2-6,0)$ & 0,5 \\
\hline Presença de complicações do DM** & $51(73,9 \%)$ & $80(58,8 \%)$ & 0,02 \\
\hline Uso de medicamentos para DM & $60(88,2 \%)$ & $112(83,6 \%)$ & 0,3 \\
\hline Atividade física & & & 0,09 \\
\hline Ativo/Pouco ativo & $52(75,4 \%)$ & $86(63,7 \%)$ & \\
\hline Sedentário & $17(24,6 \%)$ & $49(36,3 \%)$ & \\
\hline $\operatorname{IMC}^{\star \star \star}\left(\mathrm{kg} / \mathrm{m}^{2}\right)$ & $31,4(30,1-32,7)$ & $30,1(29-31,2)$ & 0,1 \\
\hline História familiar de DM (pais/irmãos) & $48(69,6 \%)$ & $82(60,3 \%)$ & 0,2 \\
\hline Saúde autopercebida como MUITO BOA e BOA & $45(66,2 \%)$ & $76(55,9 \%)$ & 0,1 \\
\hline Grau de bem-estar familiar MUITO BOM e BOM & $52(76,5 \%)$ & $109(80,1 \%)$ & 0,5 \\
\hline \multicolumn{4}{|l|}{ Utilização do serviço de saúde } \\
\hline Tipo de serviço de saúde & & & 0,05 \\
\hline Público & $48(69,6 \%)$ & $80(58,8 \%)$ & \\
\hline Privado & $21(30,4 \%)$ & $56(41,2 \%)$ & \\
\hline Número de consultas no serviço referido (último ano) & $8,5(5,9-11,1)$ & $4,9(4,1-5,7)$ & 0,009 \\
\hline Tempo que consulta no serviço (>2 anos) & $62(91,2 \%)$ & $106(78,5 \%)$ & 0,04 \\
\hline Total & 69 & 136 & \\
\hline
\end{tabular}

${ }^{*}$ Teste $t$ de Student para as médias e teste de quiquadrado de Pearson para as proporções. ${ }^{\text {**} C o m p l i c a c ̧ o ̃ e s ~ d o ~ D M: ~ n e f r o p a t i a ~ d i a b e ́ t i c a, ~ r e t i n o p a t i a ~ d i a b e ́ t i c a, ~}$ amputação de membros, infarto miocárdico, acidente vascular cerebral, coma diabético e hipoglicemia. ${ }^{* \star}{ }^{*} \mid M C$ : Índice de Massa Corporal. 
em complicaçôes do DM (73,9\% vs 58,8\%, p=0,02), em relação aos de Baixo Escore. Na utilização dos serviços, o número de consultas no último ano era maior entre o grupo de Alto Escore $(8,5$ vs 4,9, $p=0,009)$, além de uma fração maior dos pacientes estar vinculada aos serviços há 2 ou mais anos $(91,2 \%$ vs $78,5 \% ; p=0,04)$. Dos serviços com forte orientação à APS, $69,6 \%$ eram públicos, enquanto apenas $58,8 \%$ dos classificados como sendo de fraca orientação eram públicos $(\mathrm{p}=0,04)$. Tanto a média da HbAlc quanto o percentual de entrevistados com ótimo controle glicêmico $(\mathrm{HbA1c}<7)$ não apresentaram diferenças significativas entre os serviços pesquisados.

Fazendo referência ao processo de atenção e à qualidade do manejo ao DM, com a adoção de práticas baseadas em sólidas evidências e orientaçôes preventivas relevantes às principais causas de morbimortalidade do DM, a Tabela 2 apresenta diferenças significativas entre os serviços com Alto e Baixo Escore, principalmente em relação ao cuidado com os pés, aferição da PA e orientação para prática de atividade física. Por exemplo, a fração de pacientes recebendo examinação dos pés e orientaçóes sobre seus cuidados eram o dobro nos serviços de Alto Escore Geral do que naquelas recebendo esses cuidados em serviços de Baixo Escore geral.

Tabela 2. Distribuição de indicadores de qualidade do processo de atenção e práticas preventivas entre os portadores de diabetes classificados com Alto e Baixo Escore Geral de APS, Porto Alegre, 2007.

\begin{tabular}{|c|c|c|c|}
\hline Características & Alto Escore Geral da APS $(\geq 6,6)$ & Baixo Escore Geral da APS $(<6,6)$ & $\mathrm{p}^{*}$ \\
\hline Processo de atenção ao DM (n) & $(\%)$ & $(\%)$ & \\
\hline Exame dos pés§ (205) & 43,5 & 22,1 & $<0,001$ \\
\hline Orientações sobre cuidados dos pés§ (204) & 66,7 & 33,3 & $<0,001$ \\
\hline Consulta com oftalmologista ${ }^{\S}(203)$ & 46,4 & 32,1 & 0,06 \\
\hline Consulta com dentista ${ }^{\S}(205)$ & 18,8 & 13,2 & 0,3 \\
\hline PA aferida ${ }^{\S}(203)$ & 94,1 & 82,2 & 0,04 \\
\hline PA aferida última consulta (200) & 95,6 & 89,3 & 0,08 \\
\hline Cintura medida na última consulta (199) & 15,9 & 17,7 & 0,7 \\
\hline Peso medido na última consulta (200) & 75,4 & 68,7 & 0,4 \\
\hline Altura medida alguma vez (200) & 68,1 & 51,1 & 0,005 \\
\hline Exame de urina solicitado ${ }^{\S}(200)$ & 78,3 & 66,4 & 0,05 \\
\hline ECG de repouso solicitado§ $(200)$ & 44,9 & 45,8 & 0,9 \\
\hline Perfil lipídico solicitado $(200)$ & 91,3 & 75,6 & 0,004 \\
\hline \multicolumn{4}{|l|}{ Práticas/Orientações preventivas } \\
\hline Prática de atividade física ${ }^{\S}$ (203) & 79,4 & 54,8 & 0,003 \\
\hline Alimentação saudáve|§ (203) & 91,2 & 68,1 & $<0,001$ \\
\hline Higiene bucal§ (201) & 53,7 & 20,1 & $<0,001$ \\
\hline Vacina contra influenza ${ }^{\S}(203)$ & 45,6 & 24,4 & 0,01 \\
\hline Total & 69 & 136 & \\
\hline
\end{tabular}

*Teste de quiquadrado de Pearson. $\S=$ nos últimos 12 meses.

$\mathrm{Na}$ Tabela 3, são apresentadas as associaçóes não ajustadas e ajustadas de ser atendido em um serviço com alto grau de orientaçáo à APS com algumas práticas e orientaçóes preventivas fundamentais ao adequado manejo do DM. Aferiçáo da PA na última consulta, solicitação de perfil lipídico, exame e orientação para cuidados com os pés, além das recomendaçóes à prática de atividade física mantiveram-se estatisticamente significativas após controle dos confundimentos. Em geral, as associaçóes eram um pouco maiores após os ajustes.

Tabela 3. Efeito dos serviços de saúde com Alto Escore de atenção primária à saúde no processo de atenção aos portadores de DM: regressão de Poisson com variância robusta, Porto Alegre, 2007.

\begin{tabular}{|c|c|c|c|c|}
\hline Variáveis Dependentes/Desfechos & RP não ajustada (IC 95\%) & $p$ & RP ajustada ${ }^{1}$ (IC 95\%) & $p$ \\
\hline PA aferida na última consulta* & $1,07(0,99-1,15)$ & 0,06 & $1,07(1,01-1,14)$ & 0,05 \\
\hline Orientação para fazer exercícios físicos & $1,45(1,18-1,78)$ & $<0,001$ & $1,50(1,21-1,86)$ & $<0,001$ \\
\hline Orientação para fazer dieta saudável & $1,33(1,17-1,53)$ & $<0,001$ & $1,37(1,18-1,59)$ & $<0,001$ \\
\hline Exame dos pés & $1,97(1,45-2,68)$ & $<0,001$ & $2,08(1,54-2,81)$ & $<0,001$ \\
\hline Orientação sobre cuidado com os pés & $2,00(1,55-2,58)$ & $<0,001$ & $2,02(1,56-2,61)$ & $<0,001$ \\
\hline Consulta oftalmológica no último ano & $1,44(0,99-2,11)$ & 0,1 & $1,49(1,04-2,12)$ & 0,03 \\
\hline Perfil lipídico solicitado no último ano & $1,21(1,08-1,35)$ & 0,001 & $1,23(1,09-1,39)$ & 0,001 \\
\hline Exame de urina realizado no último ano & $1,18(1,01-1,37)$ & 0,03 & $1,21(1,04-1,41)$ & 0,01 \\
\hline
\end{tabular}

${ }^{1}$ ajustado para sexo, idade, escolaridade, classe econômica, cor da pele e tipo de serviço. *PA= pressão arterial. 


\section{Discussão}

Os resultados das análises multivariáveis sugerem que serviços com alto grau de orientação para a APS fornecem mais práticas e orientaçóes preventivas relevantes ao manejo das complicaçóes decorrentes do DM e atendem, de forma continuada, pacientes com doença mais avançada. Além disso, nos serviços públicos, a maior fração dos pacientes avaliou estes locais como sendo de mais alta qualidade, nos aspectos relacionados à APS, que a dos serviços particulares.

Em nosso estudo, a proporção de portadores de DM com ótimo controle da HbA1c (35,6\%) apresentou melhores resultados do que em outras pesquisas nacionais e internacionais. Estudo multicêntrico da Fundação Osvaldo Cruz (FIOCRUZ) $^{30}$ evidenciou adequado controle glicêmico em apenas $24 \%$ dos portadores de DM em nosso país. Nos EUA, estudos $^{31}$ demonstraram que cerca de $35 \%$ dos adultos com DM atingiram níveis de HbA1c $<7 \%$. Entretanto, estudos realizados em países com melhor organização da APS (Espanha e Portugal) 32,33, evidenciaram um bom controle glicêmico em $54,8 \%$ e $51,7 \%$ dos pacientes, respectivamente. Nesse contexto, é importante também lembrar que o alvo de $7 \%$ não está indicado para muitos pacientes ${ }^{34}$.

Os serviços identificados como sendo de alto grau de orientação à APS apresentaram populaçóes significativamente distintas em relação ao estado de saúde, com maior tempo de doença (10,9 anos), maior presença de complicaçóes relacionadas ao DM $(73,9 \%)$, além de terem utilizado com maior frequência os serviços de saúde ( 8,5 consultas/ano). Apesar deste cenário adverso, o controle da HbA1c não diferiu significativamente entre os serviços com alto e baixo grau de orientação à APS. Entretanto, quanto ao processo de atenção ao DM, os serviços com Alto Escore à APS apresentaram diferença inequívoca para situaçôes de prevenção e diagnóstico precoce das complicaçóes de alta morbimortalidade, principalmente prevenção de doença cardiovascular-DCV (aferição da PA, solicitação de perfil lipídico e orientação à prática de atividade física), ou da doença microvascular e neuropatias periféricas (exame e orientaçóes sobre cuidados com os pés). Estudos relacionando atributos específicos da $\mathrm{APS}^{21,35-37}$, principalmente acesso e longitudinalidade, demonstram resultados conflitantes em relaçáo à qualidade do manejo e monitoramento do DM. Em estudo realizado em Pelotas/RS, demonstrou-se uma grande variabilidade nos procedimentos terapêuticos e diagnósticos realizados pelos médicos da rede de atenção primária ${ }^{38}$. A comparação entre os resultados desse estudo com o presente demonstra melhor manejo percebido pelos usuários em Porto Alegre. Em uma revisão sistemática realizada em $2009^{39}$, pela Universidade Católica de Roma, com o objetivo de avaliar a efetividade dos instrumentos de medicina baseada em evidências (MBE) disponíveis para profissionais da APS, no manejo do DM2 foram selecionados 13 ensaios clínicos randomizados, sendo as principais intervençôes estudadas as educacionais, auditorias e baseadas em programas computacionais (lembretes e checklists). A adesão às ferramentas de MBE foi mais provável de melhorar o processo de cuidado do que os desfechos em DM. Entretanto, a utilização de programas computacionais, para acompanhamento destes pacientes, possivelmente é efetiva no manejo do DM.

Buscar a otimização dos serviços públicos e diminuir as iniquidades em saúde deve ser a meta fundamental para o SUS. Uma das formas de concretização destes objetivos é pela expansão dos serviços com alto grau de orientação para a APS. Em nosso país, a ESF ${ }^{40}$ representa este modelo de reorganização da atenção à saúde, com impacto já demonstrado na mortalidade infantil ${ }^{16}$ e nas internações por condições sensíveis à APS. Um dos desafios do SUS é reproduzir estes ótimos resultados alcançados pelas políticas de saúde materno infantis nas DCNT, em especial no DM. Aspectos importantes a serem considerados nesta transição de modelo são o apoio ao autocuidado dos pacientes, sistema de informaçôes clínicas, redesenho na oferta de consultas, utilização de diretrizes e ferramentas de auxílio às decisões, organização dos cuidados de saúde e dos recursos comunitários ${ }^{41}$. Junto a isto, a adoção de metas e objetivos bem definidos para o controle adequado de glicemia e outros fatores de risco para cada paciente, a prática de gestáo da clínica ${ }^{42}$ com abordagens multifacetadas, mudanças na cultura organizacional e o envolvimento intenso dos profissionais são alguns dos obstáculos a serem superados pelas políticas públicas de saúde.

Algumas limitaçóes metodológicas devem ser consideradas na interpretaçáo de nossos resultados. Primeiro, por ser um estudo transversal, nenhuma inferência causal pode ser realizada, devendo os achados serem interpretados como associaçóes ${ }^{43}$. Segundo, o PCATool-Brasil é um instrumento quantitativo, o que pode levar à perda de algumas dimensóes subjetivas do cuidado, melhor captadas por metodologias qualitativas. Além disso, no presente estudo, avaliamos a experiência do usuário, sem levar em consideração o papel do gestor e a experiência dos profissionais de saúde.

Em conclusão, serviços de APS avaliados como sendo de alta qualidade ofereceram, com maior frequência, cuidados preventivos básicos aos seus pacientes com diabetes, além de atenderem pacientes de maior complexidade. 


\section{Referências}

1. Andrade LOM, Bueno ICHC, Bezerra RC. Atenção Primária à Saúde e Estratégia Saúde da Família. In: Campos GWS, Minayo MCS, Akerman M, Drumond M Jr, Carvalho YM, editors. Tratado de Saúde Coletiva. São Paulo: Hucitec; Rio de Janeiro: Fiocruz; 2006. p. $783-836$.

2. Starfield B. Atenção primária: equilíbrio entre necessidades de saúde, serviços e tecnologia. Brasília: Ministério da Saúde; 2002.

3. Macinko J, Starfield B, Shi L. The contribution of primary care systems to health outcomes within Organization for Economic Cooperation and Development (OECD) countries, 1970-1998. Health Serv Res 2003 Jun; 38(3): 831-65. PMid:12822915 PMCid:PMC1360919. http://dx.doi. org/10.1111/1475-6773.00149

4. Shi L, Starfield B, Kennedy B, Kawachi I. Income inequality, primary care, and health indicators. J Fam Pract. 1999 Apr; 48(4): 275-84. PMid:10229252.

5. Shi L, Starfield B, Politzer R, Regan J. Primary care, self-rated health, and reductions in social disparities in health. Health Serv Res. 2002 Jun; $37(3)$ : 529-50. PMid:12132594 PMCid:PMC1434650. http://dx.doi.org/10.1111/1475-6773.t01-1-00036

6. Starfield B. Primary Care: concept, evaluation and policy. New York: Oxford University Press; 1992. PMCid:PMC1809870.

7. Cassady CE, Starfield B, Hurtado MP, Berk RA, Nanda JP, Friedenberg LA. Measuring consumer experiences with primary care. Pediatrics. 2000 Apr; 105(4 Pt 2): 998-1003. PMid:10742362.

8. Shi L, Starfield B, Jihaong X. Validating the Adult Primary Care Assessment Tool. J Family Practice. 2001; 50:161-75.

9. Medronho RA. Epidemiologia. São Paulo; 2003.

10. Duncan BB, Schmidt MI, Giugliani ERJ. Medicina Ambulatorial: Condutas de Atenção Primária Baseadas em Evidências. 3. ed. Porto Alegre: Artmed; 2004.

11. Brasil. Ministério da Saúde, DATASUS. Informações de Saúde. Estatísticas Vitais - Mortalidade e Nascidos Vivos. [acesso em 2006 Dez 9]. Disponível em: http://w3.datasus.gov.br/datasus/datasus php?area=359A1B378C5D0E0F359G22H0I1Jd5L25M0N\&VInclude=/site/infsaude.php

12. Venkat Narayan KM, et al. Diabetes: The Pandemic and Potential Solutions. In: Jamison DT, Breman JG, Measham AR, Alleyne G, Claeson M, Evans DB, et al., editors. Disease Control Priorities in Developing Countries. 2nd ed. New York: Oxford University Press; 2006. p. 591-604.

13. Brasil. Ministério da Saúde. Secretaria de Atenção à Saúde. Departamento de Atenção Básica. Diabetes Mellitus. Brasília: Ministério da Saúde; 2006. Cadernos de Atenção Básica. Ref Type: Serial (Book,Monograph).

14. Brasil. Ministério da Saúde.Secretaria de Políticas Públicas. HIPERDIA - Plano de reorganização da atenção à hipertensão arterial e ao diabetes mellitus. Rev Saúde Pública. 2001; 35(6): 585-8. PMid:11799474.

15. Brasil. Ministério da Saúde. Secretaria de Atenção à Saúde. Departamento de Atenção Básica. Histórico da cobertura do PSF. [acesso em 2007 June 16]. Disponível em: http://dtr2004.saude.gov.br/dab/abnumeros.php\#historico

16. Macinko J, Guanais FC, de Fátima M, de Souza M. Evaluation of the impact of the Family Health Program on infant mortality in Brazil, 1990-2002. J Epidemiol Community Health. 2006 Jan; 60(1): 13-9. PMid:16361449 PMCid:PMC2465542. http://dx.doi.org/10.1136/jech.2005.038323

17. American Diabetes Association. Standards of medical care in diabetes. Diabetes Care. 2005; 28(Suppl 1).

18. International Diabetes Federation. Clinical Guidelines Task Force. Global guideline for Type 2 diabetes. Brussels: International Diabetes Federation; 2005. Ref Type: Generic.

19. Parchman ML, Burge SK. Continuity and quality of care in type 2 diabetes: a Residency Research Network of South Texas study. J Fam Pract. 2002 Jul; 51(7): 619-24. PMid:12160500.

20. Ovhed I, Johansson E, Odeberg H, Rastam L. A comparison of two different team models for treatment of diabetes mellitus in primary care. Scand J Caring Sci. 2000; 14(4): 253-8. http://dx.doi.org/10.1111/j.1471-6712.2000.tb00593.x

21. Al Khaja KA, Sequeira RP, Damanhori AH. Comparison of the quality of diabetes care in primary care diabetic clinics and general practice clinics. Diabetes Res Clin Pract. 2005 Nov; 70(2): 174-82. PMid:15890429. http://dx.doi.org/10.1016/j.diabres.2005.03.029

22. Leinung MC, Gianoukakis AG, Lee DW, Jeronis SL, Desemone J. Comparison of diabetes care provided by an endocrinology clinic and a primarycare clinic. Endocr Pract. 2000 Sep; 6(5): 361-6. PMid:11141586. http://dx.doi.org/10.4158/EP.6.5.361

23. Greisinger AJ, Balkrishnan R, Shenolikar RA, Wehmanen OA, Muhammad S, Champion PK. Diabetes care management participation in a primary care setting and subsequent hospitalization risk. Dis Manag. 2004; 7(4): 325-32. PMid:15671789. http://dx.doi.org/10.1089/dis.2004.7.325

24. Harzheim E, Duncan BB, Stein AT, Cunha CR, Gonçalves MR, Trindade TG, et al. Quality and effectiveness of different approaches to primary care delivery in Brazil. BMC.Health Serv Res. 2006; 6: 1472-6963.

25. Harzheim E, Starfield B, Rajmil L, varez-Dardet C, Stein AT. [Internal consistency and reliability of Primary Care Assessment Tool (PCATool-Brasil) for child health services]. Cad Saude Publica 2006 Aug; 22(8): 1649-59. PMid:16832536. http://dx.doi.org/10.1590/S0102-311X2006000800013

26. Brasil. Ministério da Saúde. Secretaria de Atenção em Saúde. Departamento de Atenção Básica. Manual do instrumento de avaliação da atenção primária à saúde: primary care assessment tool pcatool - Brasil. Brasília: Ministério da Saúde; 2010.

27. Whitworth JA, Chalmers J: World health organisation-international society of hypertension (WHO/ISH) hypertension guidelines. Clin Exp Hypertens. 2004, 26: 747-752. PMid:15702630. http://dx.doi.org/10.1081/CEH-200032152 
28. Sacks DB, Bruns DE, Goldstein DE, Maclaren NK, McDonald JM, Parrott M. Guidelines and recommendations for laboratory analysis in the diagnosis and management of diabetes mellitus. Clin Chem. 2002 Mar; 48(3): 436-72. PMid:11861436.

29. Barros AJ, Hirakata VN. Alternatives for logistic regression in cross-sectional studies: an empirical comparison of models that directly estimate the prevalence ratio. BMC Med Res Methodol. 2003 Oct 20; 3 :21. PMid:14567763 PMCid:PMC521200. http://dx.doi.org/10.1186/1471-2288-3-21

30. Fundação Oswaldo Cruz - Fiocruz, Universidade Federal de São Paulo - UNIFESP. Diabetes e o baixo controle no Brasil. [acesso em 2007 Nov 7 ]. http://www ensp fiocruz br/radis/59/03 html

31. Jackson GL, Edelman D, Weinberger M. Simultaneous control of intermediate diabetes outcomes among Veterans Affairs primary care patients. $J$ Gen Intern Med. 2006 Oct; 21(10): 1050-6. PMid:16970554 PMCid:PMC1831633. http://dx.doi.org/10.1111/j.1525-1497.2006.00519.x

32. Mengual L, Roura P, Serra M, Montasell M, Prieto G, Bonet S. Multifactorial control and treatment intensity of type-2 diabetes in primary care settings in Catalonia. Cardiovasc Diabetol. 2010 Mar 29; 9: 14. PMid:20350315 PMCid:PMC2858123. http://dx.doi.org/10.1186/1475-2840-9-14

33. Cortez-Dias N, Martins S, Belo A, Fiuza M; VALSIM.Prevalence, management and control of diabetes mellitus and associated risk factors in primary health care in Portugal. Rev Port Cardiol. 2010 Apr; 29(4): 509-37. PMid:20734573.

34. Yudkin JS, Richter B, Gale EAM. Intensified glucose lowering in type 2 diabetes: time for a reappraisal. Diabetologia. 2010; 53: $2079-2085$. PMid:20686748. http://dx.doi.org/10.1007/s00125-010-1864-z

35. McAlister FA, Majumdar SR, Eurich DT, Johnson JA. The effect of specialist care within the first year on subsequent outcomes in 24,232 adults with new-onset diabetes mellitus: population-based cohort study. Qual Saf Health Care. 2007 Feb; 16(1): 6-11. PMid:17301194 PMCid:PMC2464930. http://dx.doi.org/10.1136/qshc.2006.018648

36. Mainous AG, III, Koopman RJ, Gill JM, Baker R, Pearson WS. Relationship between continuity of care and diabetes control: evidence from the Third National Health and Nutrition Examination Survey. Am J Public Health 2004 Jan;94(1):66-70. PMid:14713700 PMCid:PMC1449828. http://dx.doi. org/10.2105/AJPH.94.1.66

37. Gill JM, Mainous AG, III, Diamond JJ, Lenhard MJ. Impact of provider continuity on quality of care for persons with diabetes mellitus. Ann Fam Med. 2003 Sep; 1(3): 162-70 PMid:15043378 PMCid:PMC1466582. http://dx.doi.org/10.1370/afm.22

38. Assunção MC, Santos I, Gigante DP. [Diabetes mellitus at the primary health care level in Southern Brazil: structure, course of action and outcome]. Rev Saude Publica. 2001 Feb; 35(1): 88-95. PMid:11285523. http://dx.doi.org/10.1590/S0034-89102001000100013

39. de Belvis AG, Pelone F, Biasco A, Ricciardi W, Volpe M. Can primary care professionals' adherence to Evidence Based Medicine tools improve quality of care in type 2 diabetes mellitus? A systematic review. Diabetes Res Clin Pract. 2009 Aug; 85(2): 119-31 PMid:19539391. http://dx.doi. org/10.1016/j.diabres.2009.05.007

40. Brasil. Ministério da Saúde, Departamento de Atenção à Saúde, Departamento de Atenção Básica. Política Nacional de Atenção Básica. Brasília: Ministério da Saúde; 2006.

41. Bodenheimer T, Wagner EH, Grumbach K. Improving primary care for patients with chronic illness. JAMA. 2002 Oct 9; 288(14): 1775-9. PMid:12365965. http://dx.doi.org/10.1001/jama.288.14.1775

42. Scally G, Donaldson LJ. The NHS's 50 anniversary. Clinical governance and the drive for quality improvement in the new NHS in England. BMJ. 1998 Jul 4; 317(7150): 61-5. PMid:9651278 PMCid:PMC1113460. http://dx.doi.org/10.1136/bmj.317.7150.61

43. Rothman KJ, Greenland S. Modern Epidemiology. 2nd ed. LW\&W; 1998 